\title{
Heart Rate Variability Analysis by Chaotic Global Techniques in Children with Attention Deficit Hyperactivity Disorder
}

\author{
RUBENS WAJNSZTEJN, ${ }^{1}$ TATIANA DIAS DE CARVALHO, ${ }^{2,3}$ DAVID M. GARNER, ${ }^{4}$ LUIZ \\ CARLOS MARQUES VANDERLEI, ${ }^{5}$ MOACIR FERNANDES GODOY, ${ }^{6}$ RODRIGO DAMINELLO \\ RAIMUNDO, ${ }^{3}$ CELSO FERREIRA, ${ }^{2}$ VITOR E. VALENTI, ${ }^{7}$ AND LUIZ CARLOS DE ABREU ${ }^{3}$
}

${ }^{1}$ Núcleo Especializado em Aprendizagem, Faculdade de Medicina do ABC, FMABC, Santo André, Brazil; ${ }^{2}$ Departamento de Medicina, Disciplina de Cardiologia, Universidade Federal de São Paulo, UNIFESP, São Paulo, Brazil; ${ }^{3}$ Laboratório de Escrita Científica da Faculdade de Medicina do ABC, FMABC, Santo André, Brazil; ${ }^{4}$ Cardiorespiratory Research Group, Department of Biological and Medical Sciences, Faculty of Health and Life Sciences, Oxford Brookes University, Gipsy Lane, Oxford OX3 OBP, United Kingdom; ${ }^{5}$ Departamento de Fisioterapia da Faculdade de Ciências e Tecnologia, Universidade Estadual Paulista, UNESP, Presidente Prudente, São Paulo, Brazil; ${ }^{6}$ Núcleo Transdisciplinar de Estudos do Caos e da Complexidade. Faculdade de Medicina de São José de Rio Preto, FAMERP, São José do Rio Preto, Brazil; and ${ }^{7}$ Departamento de Fonoaudiologia da Faculdade de Filosofia e Ciências, Universidade Estadual Paulista, UNESP, Marília, Brazil

Received 26 February 2015; revised 6 May 2015; accepted 7 May 2015

\begin{abstract}
Attention deficit hyperactivity disorder (ADHD) is characterized by decreased attention span, impulsiveness, and hyperactivity. Autonomic nervous system imbalance was previously described in this population. We aim to compare the autonomic function of children with ADHD and controls by analyzing heart rate variability (HRV). Children with ADHD (22 boys, mean age 9.964 years) and 28 controls (15 boys, mean age 9.857 years) rested in supine position with spontaneous breathing for 20 min. Heart rate was recorded beat by beat. HRV analysis was performed by use of chaotic global techniques. ADHD promoted an increase in the chaotic forward parameter. The algorithm which applied all three chaotic global parameters was only the second optimum statistically measured by Kruskal-Wallis $(P<0.0001)$ and low standard deviations. It was also highly influential by principal component analysis with almost all variation covered by the first two components. The third algorithm which lacked the (high spectral Detrended Fluctuation Analysis) parameter performed best statistically. However, we chose the algorithm which applied all three chaotic globals due to previous studies mentioned in the text-forward and inverse problems. Comparison of the autonomic function by analyzing HRV with chaotic global techniques suggests an increase
\end{abstract}

Correspondence to: Tatiana Dias de Carvalho; Departamento de Medicina, Disciplina de Cardiologia, Universidade Federal de São Paulo, UNIFESP, São Paulo, Brazil. E-mail: carvalho.td1@gmail.com

The authors declare that there is no conflict of interests regarding the publication of this article 
in chaotic activity in children with ADHD in relation to the control group. (C) 2015 Wiley Periodicals, Inc. Complexity

21: 412-419, 2016

Key Words: attention deficit hyperactivity disorder; autonomic nervous system; children; heart rate variability; principal component analysis

\section{INTRODUCTION}

A ttention deficit hyperactivity disorder (ADHD) is characterized by lower levels of concentration, impulsiveness, and hyperactivity [1,2]. Evidence suggests that ADHD is more prevalent in children with cardiac diseases and drug treatment has effects on cardiovascular parameters, such as heart rate and blood pressure $[1-3]$. Considering that cardiac function is regulated by the interaction of sympathetic and parasympathetic branches of the autonomic nervous system (ANS), heart rate variability (HRV) allows the investigation of fluctuations in the intervals between consecutive heart beats (RR intervals), which are related to the influences of the ANS on the sinus node [4].

The rhythm of cardiac interbeat intervals can vary in a complex and chaotic manner [5-10]. Traditionally, methods derived from statistical mechanics have enabled researchers to investigate such systems [11]. Nonlinear methods permit the analysis of physiological processes, such as the effect of breathing on the ANS [12] and diseases, such as ADHD. A literature search revealed only one study [13] addressed the effect of ADHD on cardiac autonomic modulation using a nonlinear analysis of HRV. The aim of this study was to analyze the effect of ADHD on cardiac autonomic modulation using chaotic global [14] analysis of HRV. Furthermore, there has been speculation that if Shannon entropy $[15,16]$ and Detrended Fluctuation Analysis (DFA) [17,18] previously applied to Welch power spectra were applied to multi-taper method (MTM) spectra [19]; which are adaptive and more sensitive, the results may have greater chaotic parametric response.

In this study, we have the Electrocardiographics RR intervals in normal and ADHD children. The main objective is to take two sets of HRV time-series from control and ADHD and calculate novel parameters to achieve a significant statistical association to determine mathematically which series is the control, and which is the experimental. We are assuming that the ADHD subjects $\mathrm{RR}$ intervals behave in a nonlinear way akin to asthma [20], cardiac arrest [21,22], and epilepsy [23,24]; as dynamical conditions [25] which can be quantified and assessed by applying globally chaotic algorithms [14].

The perceived benefit for testing the correlation with HRV is that it can provide an indicator of the risk of dynamical diseases [25] in ADHD subjects. HRV is a simple, noninvasive and cheap method of monitoring the ANS. Other methods are either not sensitive enough as with Sympathetic Skin Response or too complex and expensive such as Quantiative Pupillography [26].

\section{MATERIALS AND METHODS}

\subsection{Population}

Twenty-eight children with ADHD (22 boys, mean age $9.964 \pm 1.93$ years) and 28 controls (15 boys, mean age $9.857 \pm 1.84$ years) were recruited from the Núcleo Especializado em Aprendizagem da Faculdade de Medicina do ABC (Learning Disabilities Center of Medicine ABC University), Santo André, Brazil, where they are treated by a multidisciplinary team. All volunteers in this study had the medical diagnosis of ADHD, according to the Diagnostic and Statistical Manual of Mental Disorders, 4th edition, published by the American Psychological Association [27,28], and all met the following inclusion criteria: (1) absence of congenital anomalies such as congenital heart disease, pulmonary malformations, diaphragmatic hernia, and defects of abdominal wall closure; (2) absence of central nervous system malformations and/or neurological syndromes; (3) absence of metabolic disorders; (4) absence of medication that influences cardiac autonomic modulation since that drug treatment (e.g., methylphenedite) has effects on cardiovascular parameters such as heart rate and blood pressure. The control group was composed of healthy children without a diagnosis of ADHD. Parents of the children were asked for consent. The study received approval from the Ethics Committee of Faculdade de Medicina do ABC (protocol number 312/11) and was in compliance with Resolution 196/96 of the Conselho Nacional de Saúde.

\subsection{Procedure}

Datasets were collected under controlled temperature $\left(21-23^{\circ} \mathrm{C}\right)$ and humidity (40-60\%). Participants attended the laboratory each time between 8:00 and 11:00 to avoid circadian influences. They were instructed to avoid consuming ANS stimulants for $24 \mathrm{~h}$ before evaluation. Parents and guardians of the children stayed in the room during the entire protocol. Demographics and anthropometric 
measurements were obtained following the standard recommendations proposed by Lohman et al [29]. A heart monitor strap was placed on the chest, over the distal third of the sternum, and the heart rate receiver (Polar RS800 CX monitor; Polar, Kempele, Finland) was placed on the wrist. This type of equipment has been previously validated for beat-by-beat measurements and for HRV analysis [30-32]. Children rested in supine position with spontaneous breathing for $20 \mathrm{~min}$ and were instructed to avoid talking during data collection.

For HRV analysis, the heart rate was recorded beat-bybeat at a sampling frequency of $1000 \mathrm{~Hz}$. The data series was first digitally filtered using Polar Precision Performance SW software (version 4.01.029; Polar), in which only series with more than $95 \%$ sinus rhythm beats were included [33]. It was then manually complemented, and the visual inspection of the time series on the computer showed absence of artifacts. $1000 \mathrm{RR}$ intervals were selected for data analysis.

\subsection{Statistical Analysis}

As mentioned in the introduction a potential criticism in previous studies on diabetes [34] and childhood obesity [35] with respect to chaotic global parameters is that the spectral entropy [36] and spectral Detrended Fluctuation Analysis (sDFA) [14] analysis may be more sensitive if we applied the Shannon entropy and DFA algorithms to the multi-taper spectrum [19] rather than the Welch power spectrum [37]. Thus, the spectra applied in all three chaotic global parameters would correspond.

MTM is useful for spectral estimation and signal reconstruction, of a time series of a spectrum that may contain broadband and line components. MTM is nonparametric since it does not apply an a priori, parameter dependent model of the process that generated the time series under analysis. MTM reduces the variances of spectral estimates using a small set of tapers. Data is premultiplied by orthogonal tapers created to minimize the spectral leakage owing to the finite length of the time series. A set of independent approximations of the power spectrum is calculated. Functions identified as discrete prolate spheroidal sequences (DPSS) [38] are a set of functions which optimize the tapers. They are defined as eigenvectors of a Rayleigh-Ritz minimization problem [39]. In this study, the parameters for MTM are: (i) sampling frequency of $1 \mathrm{~Hz}$; (ii) time bandwidth for the DPSS is 3; (iii) FFT length of 256; (iv) Thomson's adaptive nonlinear combination method to combine individual spectral estimates.

\subsection{Chaotic Globals}

High spectral entropy ( $h s$ Entropy) is a function of the irregularity of amplitude and frequency of the power spectrums peaks. It is derived by applying Shannon entropy to the MTM power spectrum. This output is then normalized so that the sum of the magnitude is equal to unity; giving a normalized power spectrum. We then calculate an intermediate parameter which is the median Shannon entropy of the value obtained from three different power spectra using the MTM power spectra under three test conditions: a perfect sine wave, uniformly distributed random variables, and finally the experimental oscillating signal. These values are then again normalized mathematically so that the sine wave gives a value of zero, uniformly random variables give unity, and the experimental signal between zero and unity. It is this final value that corresponds to $h s$ Entropy.

The standard DFA algorithm can be applied to datasets where statistics such as mean, variance and autocorrelation vary with time. Regarding DFA, the scaling exponent, $\alpha$ is not constant. Such variability and introduction of errors in the time-series and its mathematical relationships over the duration of the datasets is reduced by applying the algorithm to power spectra. The standard DFA derivation is shown below. Given a bounded time series $x_{t}, t \in N$ then its intergation converts it to $X_{t}$ :

$$
X_{t}=\sum_{i=1}^{t}\left(x_{i}-\left\langle x_{i}\right\rangle\right)
$$

Subsequently, $X_{t}$ is divided into windows of length $\mathrm{L}$ samples, and a local least squares straight line fit is calculated by minimising the squared error $E^{2}$ with regards to the slope and intercept parameters $a, b$ : respectively.

$$
E^{2}=\sum_{i=1}^{L}\left(X_{i}-a i-b\right)^{2}
$$

The root-mean-square divergence from the trend, is considered over every window at every time scale.

Then, the fluctuation measurement process is repeated over the whole signal at a range of different window sizes $L$, and a log-log graph of $L$ against $F(L)$ is constructed. A linear relationship $F(L) \propto L^{\propto}$ is expressed. The scaling exponent $\alpha$ is calculated as the slope of a straight line fit to the log-log graph of $L$ against $F(L)$ using least-squares.

$$
F(L)=\left[\frac{1}{L} \sum_{i=1}^{L}\left(X_{i}-a i-b\right)^{2}\right]^{\frac{1}{2}}
$$

To obtain high spectral Detrended Fluctuation Analysis ( $h s$ DFA) we calculate the spectral adaptation in exactly the same way as for $h s$ Entropy using a MTM power spectrum with the same settings; but DFA rather than Shannon entropy is the algorithm applied.

Spectral multi-taper method (sMTM) is founded on the increased intensity of broadband noise in power spectra generated by irregular and chaotic signals. SMTM is the area between the MTM power spectrum and the baseline. 


\section{TABLE 1}

The Table Below Shows the Mean Values and Standard Deviation for Chaos Forward Parameters [1 to 7] for 1000 RR Intervals from the Normal and ADHD Subjects

\begin{tabular}{lccc}
\hline Combination of Chaotic Globals & Mean and SD Normal $(n=28)$ & Mean and SD ADHD $(n=28)$ & Kruskal-Wallis $(P$-value $)$ \\
\hline CFP1 & $0.7745 \pm 0.1265$ & $0.9209 \pm 0.0916$ & $<0.0001$ \\
CFP2 & $0.5976 \pm 0.1021$ & $0.6055 \pm 0.1181$ & 0.4173 \\
CFP3 & $0.6429 \pm 0.1235$ & $0.8292 \pm 0.0859$ & $<0.0001$ \\
CFP4 & $0.6290 \pm 0.2029$ & $0.7733 \pm 0.2030$ & 0.0083 \\
CFP5 & $0.4036 \pm 0.1288$ & $0.3702 \pm 0.1594$ & 0.5498 \\
CFP6 & $0.4773 \pm 0.1448$ & $0.6733 \pm 0.1535$ & $<0.0001$ \\
CFP7 & $0.3730 \pm 0.2057$ & $0.4119 \pm 0.2251$ & 0.5943
\end{tabular}

Kruskal-Wallis test of significance was applied to results.

\subsection{Statistical Analysis}

The parameter [CFP $x$ ] represents Chaotic Forward Parameter and $x$ corresponds to the normal and ADHD datasets. There are seven different combinations of three chaotic global parameters. Since $h s$ DFA responds to chaos in the opposite way to the others, we subtract its value from unity when applying here. All three chaotic global values have equal weighting. The significances of the various combinations is assessed by multivariate analysis later. It is expected that the [CFP] which applies all three should be the most significant since it takes the information and processes it in three different ways.

$$
\begin{aligned}
& {[\mathrm{CFP} 1]=\left[\operatorname{norm}(h s \text { Entropy })^{2}+\operatorname{norm}(s \mathrm{MTM})^{2}+(1-[\operatorname{norm}(h s \mathrm{DFA})])^{2}\right]^{\frac{1}{2}}} \\
& {[\mathrm{CFP} 2]=\left[\operatorname{norm}(h s \text { Entropy })^{2}+(1-[\operatorname{norm}(h s \mathrm{DFA})])^{2}\right]^{\frac{1}{2}}} \\
& {[\text { CFP3 }]=\left[\operatorname{norm}(h s \text { Entropy })^{2}+\operatorname{norm}(s \mathrm{MTM})^{2}\right]^{\frac{1}{2}}} \\
& {[\text { CFP4 }]=\left[\operatorname{norm}(s \mathrm{MTM})^{2}+(1-[\operatorname{norm}(h s \mathrm{DFA})])^{2}\right]^{\frac{1}{2}}} \\
& {[\mathrm{CFP} 5]=\left[(1-[\operatorname{norm}(h s \mathrm{DFA})])^{2}\right]^{\frac{1}{2}}} \\
& {[\mathrm{CFP} 6]=\left[\operatorname{norm}(s \mathrm{MTM})^{2}\right]^{\frac{1}{2}}} \\
& {[\mathrm{CFP} 7]=\left[\operatorname{norm}(h s \text { Entropy })^{2}\right]^{\frac{1}{2}}}
\end{aligned}
$$

The potential pitfall in the analysis here is that the since we are only taking spectral components; the phase information is lost. Taking groupings of spectral parameters in addition to the interpeak parameters could be a rewarding exercise. Here, we undertake the same analysis as in Vanderlei [35]. Nevertheless, there are other techniques which could be added to the [CFP] such as DFA, Shannon entropy, fractal dimension [40,41], correlation dimension [42,43], approximate entropy [44], and sample entropy $[45,46]$. Note, however, that these techniques are standard techniques applied to the temporal separations of the RR-intervals. They are not chaotic globals and do not include a power spectrum step in the algorithm.

\section{RESULTS}

Parametric statistics generally assume the data are normally distributed, hence, the use of the mean as a measure of central tendancy. If we cannot normalize the data, we should not compare means. To test our assumptions of normality we apply the Anderson-Darling [47] and Lilliefors [48] tests. The Anderson-Darling test for normality applies an empirical cumulative distribution function. The Lilliefors test is an alternative algorithm which can be applied in these circumstances where the number of subjects is quite low. In the majority of cases the $P<0.05$; for both tests so we cannot assert that the observations follow a normal distribution. Therefore, we have a probability plot of mainly non-normal data and so we must apply the Kruskal-Wallis [49] test of signficance. The results illustrate that there is a wide variation in both the mean values and standard deviation for both normal and ADHD (See Table 1). The Kruskal-Wallis algorithm computes a significant statistical result for three of the seven combinations $(P<0.0001)$ for normals versus ADHD. These are combinations [CFP 1, 3, and 6].

In Figures 1 and 2, we refer to CFP1 as CFP where CFP is the summation of the derivative of three chaotic global techniques ( $h s$ Ent)(1-hsDFA)(sMTM). CFP2 to CFP4 is the summation of two such derivatives. CFP2 is (hsEnt)(1$h s$ DFA); CFP3 is ( $h s$ Ent)(sMTM) and, CFP4 is (1$h s \mathrm{DFA}$ )(sMTM). Finally CFP5 to CFP7 is the function of a sole chaotic global CFP5 is (1-hsDFA); CFP6 is (sMTM) and, CFP7 is (hsEnt). These are expressed in the equations above.

\subsection{Principal Component Analysis}

Principal Component Analysis (PCA) [50] is a multivariate technique which can be applied here (See Table 2). We have the values of CFP for seven groups of 28 subjects who are ADHD children. Hence, a grid of 7 by 28 to be 


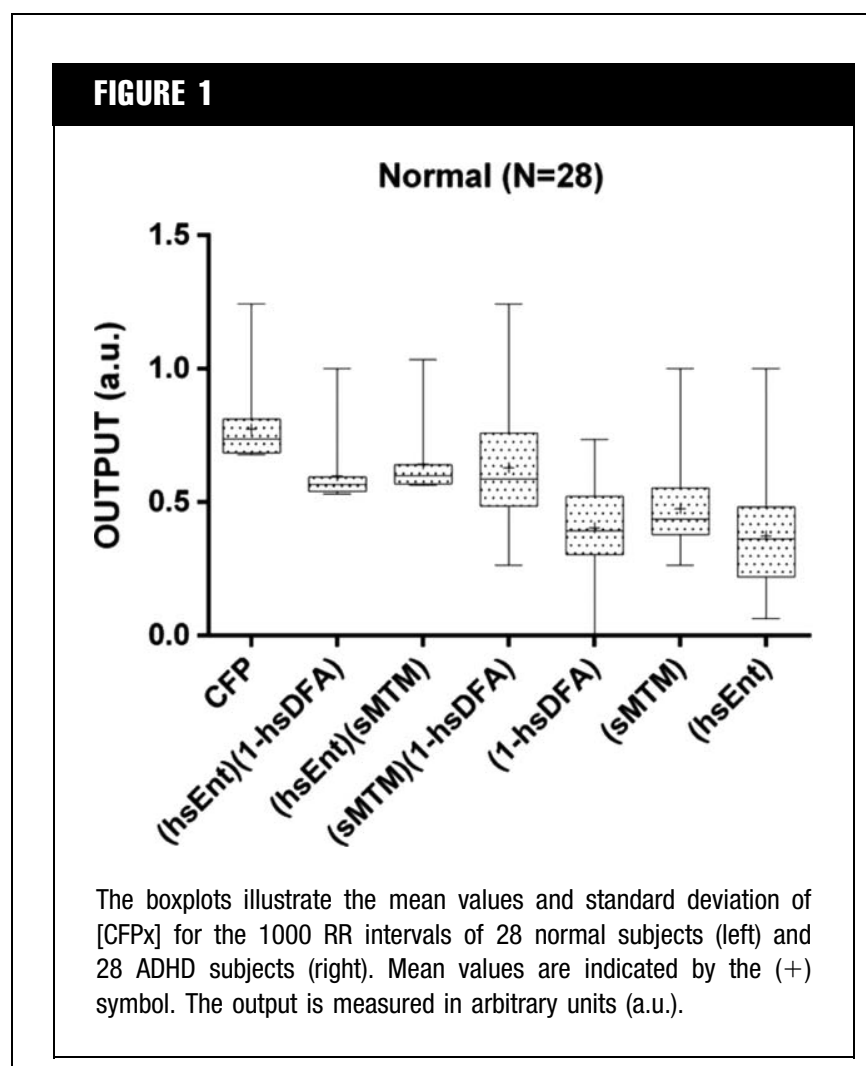

assessed. The First Principal Component (PC1) has a variance (eigenvalue) of 4.7521 and accounts for $67.9 \%$ of the total variance. The Second Principal Component (PC2) has an eigenvalue of 2.2430 accounting for $99.9 \%$ of cumulative total variance. PC2 accounting for $32.0 \%$ of its proportion of the variance. Therefore, we assume that the majortity of variance is acheived in the first two components.

[CFP 1] has the First Principal Component (0.113) and the Second Principal Component $(-0.647)$; whereas, [CFP $3]$ has the First Principal Component $(-0.151)$ and the Second Principal Component $(-0.630)$. Only the first two components need be considered due to the steep scree

\section{FIGURE 2}

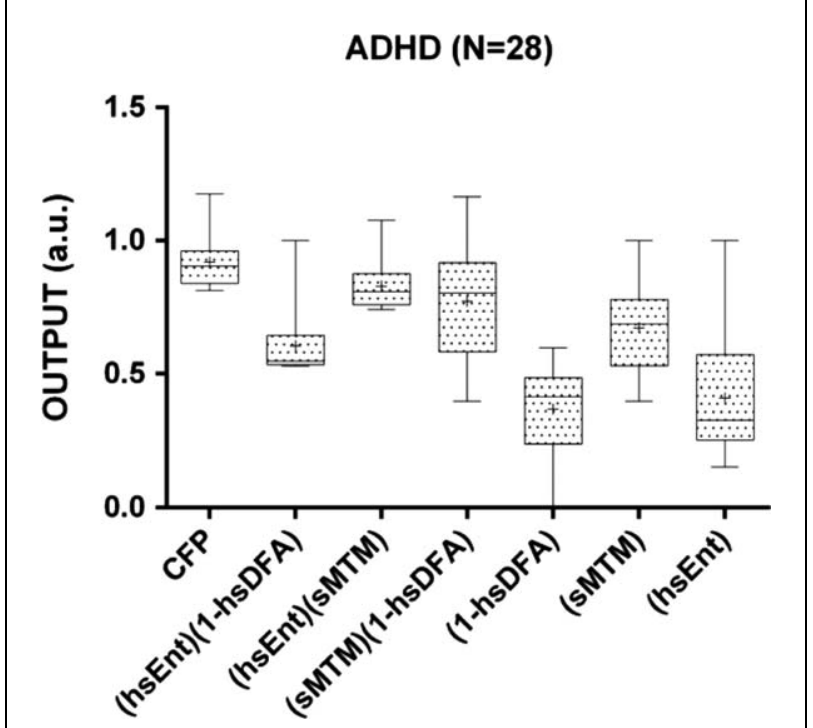

The boxplots illustrate the mean values and standard deviation of [CFPx] for the RR intervals of ADHD subjects. The number of RR intervals is 1000 and number of subjects is 28; and mean values are indicated by the $(+)$ symbol and output in arbitrary units (a.u).

plot. Only [CFP 1,3, and 6] need to be considered due to statistical signficance at the level $P<0.0001$ (Table 1). Since [CFP 6] represents just the increased intensity of broadband noise in the MTM power spectrum we do not consider it further. Accordingly, [CFP 1] which applies all three chaotic globals techniques is the best overall combination with regards to influencing the correct outcome. This on the basis of three statistical tests (See Discussion).

\section{DISCUSSION}

The ANS plays an important role in regulation of physiological mechanisms, such as cardiac and metabolic

\section{TABLE 2}

The Table Below is the Principal Component Analysis for $\left[\mathrm{CFP}_{x}\right]$ for Seven Groups of 28 Subjects Who Are ADHD Children

\begin{tabular}{lrrrrrrr}
\hline [CFP] & PC1 & PC2 & PC3 & PC4 & PC5 & PC6 & PC7 \\
\hline CFP1 & 0.113 & -0.647 & 0.517 & -0.421 & 0.259 & 0.240 & -0.020 \\
CFP2 & -0.394 & -0.343 & 0.333 & 0.674 & -0.180 & -0.049 & 0.359 \\
CFP3 & -0.151 & -0.630 & -0.584 & 0.071 & -0.005 & -0.273 & -0.400 \\
CFP4 & 0.445 & -0.161 & 0.036 & -0.220 & -0.435 & -0.605 & 0.413 \\
CFP5 & 0.458 & -0.037 & 0.233 & 0.310 & -0.519 & 0.241 & -0.558 \\
CFP6 & 0.438 & -0.196 & -0.456 & 0.190 & 0.064 & 0.551 & 0.467 \\
CFP7 & -0.458 & -0.039 & -0.134 & -0.429 & -0.661 & 0.371 & 0.109
\end{tabular}

PC1 refers to the first prinicipal component, PC2 the second up to the seventh at PC7. 
systems; and alterations in autonomic activity in children with ADHD have been investigated in several studies $[1,3,13]$. To the best of our knowledge, this is the first study to examine the effect of ADHD on cardiac autonomic modulation using chaotic global [14] analysis of HRV.

[CFP 1 and 3] are the main functions suitable as deduced by the three assessments (Kruskal-Wallis : $P$-value $<0.0001$, low standard deviation and influencial PCA). There is evidence to apply [CFP 1] as the most robust function as with the optimization study by Garner and Ling [14]. This in addition to forward problems in childhood obesity [35], diabetes mellitus [34] and COPD [51]. Nevertheless, it could be argued that [CFP 3] is the best on the basis of this study alone. With regards to PCA applied to the seven different arrangements of chaotic globals for ADHD subjects; 99.9\% of influence is achieved by the first two principal components. The third algorithm [CFP3] which lacks the (1-hsDFA) parameter, performs best on standard deviation which are low and PCA which the first two components signify a strong influence. It is important to recognize that in all cases, where the differences are significant the chaosity of the data increases from normal to ADHD children.

A literature search revealed only one study [13] addressed the effect of ADHD on cardiac autonomic modulation using a nonlinear analysis of HRV. In this investigation, according to the frequency and time domains and the Poincare plot, the indexes that indicate parasympathetic activity were higher in children with ADHD than in children without the disorder. In another study that evaluated ANS functioning and the effects of methylphenidate in stimulant-free children with ADHD and controls, the authors [1] also found that stimulant-free children with ADHD have a parasympathetic dominance of the auto- nomic balance relative to control subjects. We could suggest that increased parasympathetic is related to increased chaosity found in this work.

So, we have developed two robust functions which take short time-series of HRV and discriminate between the control and experimental groups. There is a very high level of significance for both these algorithms $(P<0.0001)$. By applying either of these novel functions to the shorter time-series via spectrally determined parameters it should be possible to determine which are ADHD or normal. The relationship between $\mathrm{ADHD}$ and complexity measures is useful in the risk assessment of dynamical diseases [25] associated with the condition. It identifies severity of the situation from a noninvasive, cheap, and reliable method of monitoring the ANS. This is useful in treatments, assisting the determination of the level of medical intervention especially in related dynamical diseases.

Future development could involve the DPSS of the MTM being adjusted to optimize the final level of significance. In addition, the weighting of the three chaotic global parameters could be modified since here they have weightings of unity. It would also be statistically favourable to have larger, but equal datasets for both normal and ADHD subjects. If the time-series were longer this may also enhance statistical significances.

\section{Conclusions}

The chaotic response of HRV in child subjects with ADHD increases. The parameter which applies all three chaotic global parameters is statistically most significant based overall on this and previously cited studies. In this case, the three chaotic global derivatives are summated and we term this function chaos forward parameter. This is useful in the risk assessment of dynamical diseases associated with ADHD.

\section{REFERENCES}

1. Negrao, B.L.; Crafford, D.; Viljoen, M. The effect of sympathomimetic medication on cardiovascular functioning of children with attention-deficit/hyperactivity disorder: Cardiovascular topic. Cardiovasc J Afr 2009, 20, 296-299.

2. Meijer, W.M.; Faber, A.; van den Ban, E.; Tobi, H. Current issues around the pharmacotherapy of ADHD in children and adults. Pharm World Sci 2009, 31, 509-516.

3. Ballard, J.E.; Boileau, R.A.; Sleator, E.K.; Massey, B.H.; Sprague, R.L. Cardiovascular responses of hyperactive children to methylphenidate. JAMA 1976, 236, 2870-2874.

4. Camm, A.J.; Malik, M.; Bigger, J.T.; Breithardt, G.; Cerutti, S.; Cohen, R.J.; Coumel, P.; Fallen, E.L.; Kennedy, H.L.; Kleiger, R.E. Heart rate variability: Standards of measurement, physiological interpretation and clinical use. Task Force of the European Society of Cardiology and the North American Society of Pacing and Electrophysiology. Circulation 1996, 93, 1043-1065.

5. Seely, A.J.; Macklem, P. Fractal variability: An emergent property of complex dissipative systems. Chaos 2012, $22,013108$.

6. Goldberger, A.L.; Rigney, D.R.; West, B.J. Chaos and fractals in human physiology. Sci Am 1990, 262, 42-49.

7. Goldberger, A.L.; West, B.J. Chaos and order in the human body. MD Comput 1992, 9, 25-34.

8. Amaral, L.A.; Diaz-Guilera, A.; Moreira, A.A.; Goldberger, A.L.; Lipsitz, L.A. Emergence of complex dynamics in a simple model of signaling networks. Proc Natl Acad Sci USA 2004, 101, 15551-15555.

9. Costa, M.; Goldberger, A.L.; Peng, C.K. Multiscale entropy analysis of biological signals. Phys Rev E Stat Nonlin Soft Matter Phys 2005, 71, 021906.

10. Elbert, T.; Rockstroh, B.; Kowalik, Z.J.; Hoke, M.; Molnar, M.; Skinner, J.E.; Birbaumer, N. Chaotic brain activity. Electroencephalogr Clin Neurophysiol Suppl 1995, 44:441-9, 441-449.

11. Ho, M.-W. The Rainbow and the Worm: The Physics of Organisms; World Scientific, Singapore, 2008. 
12. Perakakis, P.; Taylor, M.; Martinez-Nieto, E.; Revithi, I.; Vila, J. Breathing frequency bias in fractal analysis of heart rate variability. Biol Psychol 2009, 82, 82-88.

13. de Carvalho, T.D.; Wajnsztejn, R.; de Abreu, L.C.; Marques Vanderlei, L.C.; Godoy, M.F; Adami, F; Valenti, V.E.; Monteiro, C.B.; Leone, C.; da Cruz Martins, K.C.; Ferreira, C. Analysis of cardiac autonomic modulation of children with attention deficit hyperactivity disorder. Neuropsychiatr Dis Treat 2014, 10, 613-618.

14. Garner, D.M.; Ling, B.W.K. Measuring and locating zones of chaos and irregularity. J Syst Sci Complex 2014, 27, 494-506.

15. Zyczkowski, K. Renyi extrapolation of Shannon entropy. Open Syst Inf Dyn 2003, 3, 297-310.

16. Shannon, C.E. A mathematical theory of communication. Bell Syst Tech J 1948, 27, 379-423.

17. Peng, C.K.; Havlin, S.; Stanley, H.E.; Goldberger, A.L. Quantification of scaling exponents and crossover phenomena in nonstationary heartbeat time series. Chaos 1995, 5, 82-87.

18. Donaldson, G.C.; Seemungal, T.A.; Hurst, J.R.; Wedzicha, J.A. Detrended fluctuation analysis of peak expiratory flow and exacerbation frequency in COPD. Eur Respir J 2012, 40, 1123-1129.

19. Percival, D.B.; Walden, A.T. Spectral Analysis for Physical Applications: Multitaper and Conventional Univariate Techniques; Cambridge University Press, New York, NY, USA 1993.

20. Que, C.L.; Kenyon, C.M.; Olivenstein, R.; Macklem, P.T.; Maksym, G.N. Homeokinesis and short-term variability of human airway caliber. J Appl Physiol 2001, 91, 1131-1141.

21. Goldberger, A.L.; Rigney, D.R.; Mietus, J.; Antman, E.M.; Greenwald, S. Nonlinear dynamics in sudden cardiac death syndrome: Heartrate oscillations and bifurcations. Experientia 1988, 44, 983-987.

22. Goldberger, A.L.; Peng, C.K.; Lipsitz, L.A. What is physiologic complexity and how does it change with aging and disease? Neurobiol Aging 2002, 23, 23-26.

23. Skinner, J.E.; Molnar, M.; Vybiral, T.; Mitra, M. Application of chaos theory to biology and medicine. Integr Physiol Behav Sci 1992, 27, 39-53.

24. Stead, M.; Worrell, G.A.; Litt, B. Frequency and dependence of long range temporal correlations in human hippocampal energy fluctuations. Complexity 2005, 10, 35-44.

25. Mackey, M.C.; Milton, J.G. Dynamical diseases. Ann N Y Acad Sci 1987, 504, 16-32.

26. Baum, P.; Petroff, D.; Classen, J.; Kiess, W.; Bluher, S. Dysfunction of autonomic nervous system in childhood obesity: A cross-sectional study. PLoS One 2013, 8, e54546.

27. Park, S.; Kim, J.W.; Yang, Y.H.; Hong, S.B.; Park, M.H; Kim, B.N.; Shin, M.S.; Yoo, H.J.; Cho, S.C. Possible effect of norepinephrine transporter polymorphisms on methylphenidate-induced changes in neuropsychological function in attention-deficit hyperactivity disorder. Behav Brain Funct 2012, 8, 22.

28. Cantwell, D.P. Attention deficit disorder: A review of the past 10 years. J Am Acad Child Adolesc Psychiatry 1996, 35, 978-987.

29. Lohman, T.G.; Roche, A.F; Martorell, R. (eds). Anthropometric standardization reference manual. Human Kinetics 1988, Champaign: 177p

30. Vanderlei, L.C.M.; Silva, R.A.; Pastre, C.M.; Azevedo, F.M.; Godoy, M.F. Comparison of the Polar S810i monitor and the ECG for the analysis of heart rate variability in the time and frequency domains. Braz J Med Biol Res 2008, 41, 854-859.

31. Gamelin, F.X.; Berthoin, S.; Bosquet, L. Validity of the polar S810 heart rate monitor to measure R-R intervals at rest. Med Sci Sports Exerc 2006, 38, 887-893.

32. Gamelin, F.X.; Baquet, G.; Berthoin, S.; Bosquet, L. Validity of the polar S810 to measure R-R intervals in children. Int J Sports Med 2008, 29, 134-138.

33. Godoy, M.F.; Takakura, I.T.; Correa, P.R. Relevância da análise do comportamento dinâmico não linear (Teoria do Caos) como elemento prognóstico de morbidade e mortalidade em pacientes submetidos à cirurgia de revascularização miocárdica. Arq Ciênc Saúde 2005, 12, 167-171.

34. De Souza, N.M.; Vanderlei, L.C.M.; and Garner, D.M. Risk evaluation of diabetes mellitus by relation of chaotic globals to HRV. Complexity 2015, 20, 84-92.

35. Vanderlei, F.M.; Vanderlei, L.C.; Garner, D.M. Chaotic global parameters correlation with heart rate variability in obese children. J Human Growth Dev 2014, 24, 24-30.

36. Johnson, R.; Shore, J. Which is the better entropy expression for speech processing:-S log S or log S? IEEE Trans Acoust Speech Signal Process 1984, 32, 129-137.

37. Alkan, A.; Kiymik, M.K. Comparison of AR and Welch methods in epileptic seizure detection. J Med Sys 2006, 6, 413-419.

38. Slepian, S. Prolate spheroidal wave functions, Fourier analysis and uncertainty, V, The discrete case. Bell Syst Tech J 1978, 57, $1371-1430$.

39. Gould, S.H. Variational Methods for Eigenvalue Problems: An Introduction to the Methods of Rayleigh, Ritz, Weinstein, and Aronszajn; Courier Dover Publications, 1995.

40. Anier, A.; Lipping, T.; Melto, S.; Hovilehto, S. Higuchi fractal dimension and spectral entropy as measures of depth of sedation in intensive care unit. Conf Proc IEEE Eng Med Biol Soc 2004, 1, 526-529.

41. Shono, H.; Peng, C.K.; Goldberger, A.L.; Shono, M.; Sugimori, H. A new method to determine a fractal dimension of nonstationary biological time-serial data. Comput Biol Med 2000, 30, 237-245.

42. Burioka, N.; Cornelissen, G.; Halberg, F; Kaplan, D.T. Relationship between correlation dimension and indices of linear analysis in both respiratory movement and electroencephalogram. Clin Neurophysiol 2001, 112, 1147-1153.

43. Van Leeuwen, P.; Bettermann, H.; An der, Heiden U.; Kummell, H.C. Circadian aspects of apparent correlation dimension in human heart rate dynamics. Am J Physiol 1995, 269, H130-H134. 
44. Pincus, S.M.; Goldberger, A.L. Physiological time-series analysis: What does regularity quantify? Am J Physiol 1994, 266, H1643-H1656.

45. Chen, Y.; Pham, T.D. Sample entropy and regularity dimension in complexity analysis of cortical surface structure in early Alzheimer's disease and aging. J Neurosci Methods 2013, 215, 210-217.

46. Eduardo Virgilio, S.L.; Otavio, M.L. Evaluation of physiologic complexity in time series using generalized sample entropy and surrogate data analysis. Chaos 2012, 22, 043105.

47. Anderson, T.W.; Darling, D.A. A test of goodness of fit. J Am Stat Assoc 1954, 49, 765-769.

48. Razali, N.M.; Wah, Y.B. Power comparisons of shapiro-wilk, kolmogorov-smirnov, lilliefors and anderson-darling tests. J Stat Model Anal 2011, 2, 21-33.

49. Kruskal, W.H.; Wallis, W.A. Use of ranks in one-criterion variance analysis. J Am Stat Assoc 1952, 260, 583-621.

50. Jolliffe, I.T. Principal Component Analysis, Series: Springer Series in Statistics; Springer, New York, 2002.

51. Bernardo, A.F.B.; Vanderlei, L.C.M.; Garner, D.M. HRV Analysis: A clinical and diagnostic tool in Chronic Obstructive Pulmonary Disease; International Scholarly Research Notices 2014; 673232:6. 